\title{
Una réplica al empresario innovador schumpeteriano
}

\section{A reply to the innovative Schumpeterian entrepreneur}

\author{
Alexander Quintero B. \\ Universidad Surcolombiana, Colombia \\ alexanderquintero@mialegria.edu.co \\ Rafael A. Méndez L \\ Universidad Surcolombiana, Colombia \\ ramendez56@hotmail.com \\ Rolando Centeno T. \\ Universidad Surcolombiana \\ rolandzenden@hotmail.com
}

\section{Introducción}

El lector podrá encontrar el título del presente ensayo algo ambicioso. Tal vez lo sea, desde la intención al menos, ya que se pretende plantear algunos cuestionamientos al uso de los aportes teóricos de Schumpeter, específicamente del concepto de innovación, por parte de los investigadores de la historia empresarial, en particular, aquellosquese preguntanenelcontexto colombiano por el origen de los empresarios. El reto es poder esbozar algunos posibles caminos para superar tales cuestionamientos.

El ensayo surge como respuesta a un interrogante que se plantean los autores acerca del origen de los empresarios en una región periférica de Colombia. Con la reflexión teórica se coligió que para calificarlos como tales, estos sujetos debían ser enmarcados en algo más que el agente tradicional que combina recursos o genera beneficios ${ }^{1}$. Desde las ciencias sociales se identifican, al menos, tres enfoques para abordar este objeto de investigación. Primero, aquel que asocia la formación empresarial a un marco institucional existente. Segundo, la perspectiva sociológica, que en los últimos años tiende, además de los valores, a incluir la variable redes sociales. En tercer lugar, abundan los trabajos que vinculan el origen de la iniciativa empresarial a las características o rasgos de la personalidad; esto implica una revisión desde las ciencias del comportamiento.

En particular, se advierte que el empresario como objeto de estudio ha llegado a ser tema atractivo para los economistas neoclásicos. Desde esta perspectiva se vincula la acción empresarial con la asunción de riesgos y la actuación bajo incertidumbre (Knight, 1947), la detección y aprovechamiento de oportunidades (Kirzner, 1979), pero sobre todo, el agente económico calificado como empresario es referido al promotor de desequilibrios a través de la innovación (Schumpeter, 1997).

Estudios recientes se han focalizado en la formación del empresariado en economías orientadas en losúltimos años hacia el capitalismo como China y Rusia (Djankov et al., 2005, 2006). También los estudios de género vinculados a la formación empresarial dan cuenta de éste fenómeno(Paré\&Therasme, 2010).Aún,elmismo concepto de empresario causa conflicto; tal como lo aduce Zen \& Fracasso (2008) al calificarlo de dinámico y multifacético, influenciado por

1 En economía el término se utilizado para designar la ganancia que se obtiene de un proceso o actividad económica. 
las revoluciones tecnológicas y sociales. En el contexto colombiano se destacan los trabajos de Safford (1977, 1989), Molina Londoño (1998, 2003), Arroyo (2006), Albeiro y Alonso Llanos (Dávila, 2003), Quintero y Centeno (2007), para citar algunos, los cuales abordan la formación o el origen de una clase empresarial en diversas regiones del país. Dentro de este contexto, se ha propuesto la categoría de negociante que difiere de la concepción de empresario en la medida que designa la conducta económica: el patrón de especialización-diversificación, como sus nexos con la política y el estado (Dávila, 1986).

Para cumplir con el propósito, se estructuró el escrito en cinco partes. Inicialmente se presenta un panorama general del estado de la historia empresarialenColombia.Seguidamenteserevisan las contribuciones posteriores efectuadas en torno al concepto de innovación, para exponer luego 2 estudios de caso de empresarios. Posteriormente se relacionan los elementos centrales del concepto de innovación para finalmente concluir con las lecciones aprendidas dejando para la discusión una propuesta que considere la inclusión desde la perspectiva de la acción social.

\section{La historia empresarial colombiana}

El estudio del empresariado colombiano es reciente dentro de la historiografía. Éste se remonta a unos primeros trabajos en los 70 para un aumento gradual a partir de los 90 . A pesar de que se aprecia un campo en formación con abordajes desde perspectivas académicas diferentes a la historia oficial y conmemorativa, aun "hay un largo camino por recorrer" para que se incorporen los aportes de las ciencias sociales dentro de los recursos de investigación histórica. También prevalece el trabajo individual y aislado, con escasa y esporádica crítica académica (Dávila, 2003). Con una mínima vinculación a la comunidad académica internacional de la Business History y con recientes programas de doctorados y la creación de la asociación de historiadores económicos que se convierten en la esperanza para el desarrollo de una comunidad.

En resumen, la investigación en historia empresarial tiende a ser de carácter artesanal y monográfico (Colmenares, 1990), citado por Dávila (2003), con una labor documental de diferentes grados de rigor historiográfico, sin apoyo, por lo general, en referentes teóricos explícitos y sin el objetivo de confrontar hipótesis generales.

\section{¿Quién es el empresario en la historiografía colombiana?}

El empresario como objeto de estudio en Colombia, aunque tímidamente, es un tema que ha sido abordado desde perspectivas teóricas de la economía neoclásica, superando la "simple" definición de agente que combina recursos y diferenciándolo del capitalista, e incluso del comercianteydelnegociante.Desdeestaperspectiva se vincula la acción empresarial con la asunción de riesgos y la actuación bajo incertidumbre (Knight, 1947), la detección y aprovechamiento de oportunidades (Kirzner, 1979), pero sobre todo, el agente económico calificado como empresario es referido al promotor de desequilibrios a través de la innovación (Schumpeter, 2002).

Es preciso anotar, que en términos amplios, la teoría neoclásica de la economía no le dio importancia al estudio del empresario y de las empresas. Los escasos conceptos empleados para definir la acción empresarial provienen desde los clásicos. Son categorías que al parecer arrojan luz en un contexto de exiguo pensamiento. En conclusión, desde la economía no se advierten enfoques que permitan reflexionar la realidad que producen los empresarios.

En Knight (1947) el empresario hace previsiones futuras sobre una demanda, estas decisiones están basadas en la información y el conocimiento de las necesidades del consumidor y sus factores de producción. Sin embargo, la demanda es incierta; es decir, el empresario actúa bajo riesgo e incertidumbre, en tanto hay una aleatoriedad con probabilidades de ocurrencia conocidas (riesgo), que coexiste con la incertidumbre (aleatoriedad con probabilidades desconocidas). El beneficio se determina en función del riesgo asumido.

Para Kirzner (1979), el "empresario puro" es un autor de decisiones cuyo papel completo 
surge de estar alerta a las oportunidades hasta entonces ocultas. Se trata de un agente situado en un sistema de información imperfecta por parte de los participantes en el mercado. Así, las decisiones surgen, no simplemente del conocimiento sustantivo de los datos del mercado. El tipo de conocimiento requerido es el de "saber dónde buscar el conocimiento". Para Kirzner (1998), la palabra que capta mejor este tipo de "conocimiento" es el término perspicacia (alertness), estar alerta a oportunidades.

Por su parte Schumpeter (1997) ubicó al empresario como el promotor fundamental del desarrollo económico, el cual se logra por la introducción de nuevas combinaciones por parte del empresario. Estas nuevas combinaciones hacen referencia a la introducción de un nuevo producto o una nueva calidad de producto, un nuevo método de producción, un nuevo mercado, una nueva fuente de materias primas o productos semifacturados y finalmente la creación de una nueva organización en cualquier sector.

El concepto de innovación en Schumpeter está referido a las nuevas combinaciones mencionadas. Sin embargo, define también la innovación como "la creación de una nueva función de producción", lo cual, dio pie a serias divergencias y críticas. En Schumpeter se aprecia que la innovación se orienta hacia la aplicación práctica de conocimiento, las ideas o descubrimientos.

Así, el empresario es considerado como el único agente capaz de promover el desequilibrio o la discontinuidad, lo cual es logrado con la innovación, vale decir, llevando a cabo nuevas combinaciones. Es importante tener en cuenta que en atención a Schumpeter, los empresarios no son ni los inventores, ni los capitalistas, en tanto que la invención es calificada como un factor exógeno, siendo solamente los factores endógenos generadores del desequilibrio y por tanto del desarrollo económico. La invención es definida por Schumpeter (1939) como aquel producto o proceso que se genera en la esfera científico-técnica y que permanece en este ámbito in eternum, hasta que es superado o sustituido por una nueva invención.
En suma, siguiendo a Schumpeter, la transición de un crecimiento económico rutinario -un estado de equilibrio que se repite periódicamente- a un desarrollo económico, es logrado por el individuo con comportamiento proactivo hacia las nuevas combinaciones. El desarrollo económico implica un cambio sustancial en los ingresos de los hogares y las empresas, en el consumo y una función de producción dinamizada con la inversión, de allí su diferencia con el elemental crecimiento económico.

\section{La innovación, nuevos enfoques, otras controversias}

Para iniciar es válido reconocer que hoy en día existe un amplio consenso en cuanto a la trascendencia de la innovación en el contexto no sólo organizacional y para el desarrollo económico sino en la sociedad misma. Es el activo más significativo (Edvinson, 2008) y principal motor para crecer y mantener rentabilidad (Elmquist, 2009).

En consideración a que la innovación como concepto es un fenómeno dinámico y polisémico es válido empezar con claridad conceptual. Por ello, se esbozará la trayectoria seguida del concepto con el propósito de cotejarlo con los aportes ya expuestos de Schumpeter. Antes de avanzar, es necesario aclarar la diferencia que tiene la innovación frente al concepto de desarrollo de producto, puesto que a pesar de ciertas aproximaciones, no son lo mismo.

Una primera lectura permite entender que del modelo de desarrollo de productos se avanzó a uno de innovación. Este hecho marca también la evolución de una "vieja economía" basadas en los factores tradicionales de producción a "una nueva economía" del conocimiento (Chinying, 2001). Aún a mediados del siglo pasado se adelantaba la investigación desde el laboratorio de la empresa soportado en un modelo que Varmá (1995) calificó de autónomo. Lo esencial del laboratorio o la tradicional función de I+D era el desarrollo de productos. Entonces, en el mejor de los casos se entenderá por desarrollo de productos una transformación de una oportunidad de mercado en un producto disponible para la venta (Krishnan y Ulrich, 2000) 
Por otra parte, la innovación puede ser entendida como un proceso que abarca la creación y utilización del conocimiento para el desarrollo e introducción de algo nuevo y útil, vale decir, todo conocimiento nuevo, aplicado y aceptado (Wallin y Krogh, 2010). Por su parte, Jacobide (2006), lo enmarca como habilidad para generar nuevas soluciones, en tanto que Lundvall (2006) lo engloba en un proceso de aprendizaje colectivo. Igualmente, Lundvall (2002), define la innovación como un proceso que abarca: a) la discontinuidad en las características técnicas $o$ en el uso de un nuevo producto o proceso y b) la introducción, difusión y adaptación del nuevo artefacto. Yahiaoui (2008) lo define como una práctica de gestión vista como nueva por la organización, que afecta el funcionamiento social. Un autor de reconocida trayectoria como Edvinson (2004), conceptualiza la innovación como la reutilización de ideas y conocimientos existentes para generar nuevo conocimiento.

Al analizar el emprendimiento Orrego (2012) encuentra que la innovación tiene que ver con combinaciones de nuevas técnicas o conocimientos aplicados a la creación, adaptación o mejoramiento de productos o servicios, de tal forma que se logre un mejor desempeño y resultados económicos sostenibles. Así, la innovación es creatividad aplicada al desarrollo de nuevas ideas.

De acuerdo con la OECD y EUROSTAD (2005) la innovación es entendida como:

La introducción de un nuevo, o significativamente mejorado, producto (bien o servicio), de un proceso, de un nuevo método de comercialización, o de un nuevo método organizativo, en las prácticas internas de la empresa, la organización de lugar de trabajo o las relaciones exteriores. Para que haya innovación hace falta como mínimo que el producto, el proceso, el método de comercialización o el método de organización sean nuevos (o significativamente mejorados) para la empresa...una característica común a todos los tipos de innovación es que deben haber sido introducidos. Se dice que un nuevo producto (o mejorado) se ha introducido cuando ha sido lanzado al mercado. Se dice que un proceso, un método de comercialización o un método de organización se ha introducido cuando ha sido utilizado efectivamente en el marco de las operaciones de una empresa.

La innovación implica la utilización de un nuevo conocimiento o de una nueva combinación de conocimientos existentes ( $\mathrm{p}$. 56-57)

Siguiendo estas mismas fuentes, se identifican cuatro tipos de innovaciones: 1) de producto, 2) proceso, 3) mercadotecnia y 4) organización. Advirtiendo que toda clasificación es arbitraria, se tiene que también se organiza la innovación de acuerdo con la originalidad o el impacto en: 1) radical, 2) de ruptura y 3) incremental. Dependiendo de la fuente para la obtención de las ideas se puede ordenar en innovación abierta e innovación cerrada y finalmente según el alcance será tecnológica, organizacional, cultural y social.

Por otra parte, se tiene que en el proceso de evolución de la investigación y el desarrollo, además de virar hacia la innovación como resultado interno de una organización, se plantea hoy en día el concepto de innovación abierta. En atención a Chesbrough (2003) se tiene que la función de I+D no es un activo estratégico hoy en día. Las empresas pueden y deben utilizar no solo las ideas internas sino externas. Se trata de un conjunto de prácticas para sacar un mejor provecho de la innovación. Desde la visión de los economistas la innovación abierta tiene sentido para contrarrestar las contingencias y aprovechar los costos de transacción mediando con un contrato acciones o parte del proceso de innovar; sin embargo, la cuestión es mucho más profusa.

Admitir la necesidad de "abrir" el proceso de innovación implica hacer partícipes a los clientes, proveedores y otras partes interesadas, trasladando el interés hacia la innovación no sólo de productos sino de procesos. Esta concepción aplica para la mejora de procesos administrativos, calidad del producto, así como el acceso a conocimientos instalados fuera de la organización (Wallin y Krogh, 2010). La innovación abierta es en sí misma un proceso, lo cual conlleva a establecer las características de los insumos, o sea el tipo y fuente, así como los mecanismos de integración y transformación del conocimiento. Es una estrategia de innovación 
bajo la cual se va más allá de los límites internos y donde la cooperación es fundamental (Bigliardi, y Galati, 2013)

La innovación demanda la colaboración de personas y equipos con experiencia en diversos dominios oáreas de conocimiento, lo cual implica la integración de agentes de fuera y dentro de la organización. Siendo así, la innovación requiere crear conocimiento sobre las necesidades del cliente, las tendencias del mercado, las nuevas tecnologías y el desarrollo técnico y científico (Wallin y Krogh, 2010) (Lundvall, 2002) (Dtling, 2006). Este hecho ha llevado a plantear, incluso, el concepto de redes de innovación, en el sentido del establecimiento de relaciones de cooperación entre instituciones, empresas, en general partes interesadas (Kogut, 2000).

De lo anterior se advierte la trascendencia que tiene el entorno para la innovación, el cual provee mecanismos e instancias de interrelación, llegando a involucrar, tal como el caso de los sistemas nacionales de investigación, hasta las instancias del orden gubernamental (Ranga, Miedema y Jorna, 2008). La innovación requiere conocimiento y transferencia, y estos requerimientos se cumplen en la interacción no en el aislamiento, es decir, se relaciona del nivel de conectividad y complementariedad funcionando como ecosistema. De allí la necesidad de inscribirla en una red de relaciones, en un sistema, sea nacional, regional o local (Groenewegen, 2006).

En otro extremo y por lo cual requiere mención aparte, Taylor (1990) plantea desde la experiencia particular de un hombre de empresa que la innovación es ante todo una experiencia emocional, ya que a pesar de existir la posibilidad de enseñar lo técnico, no es posible enseñar la curiosidad, ni otros aspectos que tienen que ver con la personalidad, la educación, experiencias y las singularidades de cada persona.

En la parte final del presente artículo se analizan los diversos elementos que dan forma al concepto de innovación y que fueron advertidos en párrafos anteriores. Con ellos se construirá la réplica, en el sentido de contraponer un argumento al concepto schumpeteriano de innovación y su inclusión en la historiografía del empresario colombiano. Por lo pronto, se dilucida el elemento fundamental para la innovación: el conocimiento.

\section{La causa de la innovación: el conocimiento}

Sveiby (1997) al introducirse en este acápite encuentra que la palabra conocimiento tiene varias acepciones. Entreéstas se puede mencionar: información, toma de conciencia, saber, cognición, ciencia, experiencia, competencia, pericia, habilidad práctica, aprendizaje, sabiduría, certeza. Por tanto y parodiando a Ross (1996) intentar comprender las implicaciones de la innovación demanda entender primero la naturaleza del conocimiento. Siendo así, las siguientes líneas intentan esbozar el asunto para el propósito de la presente reflexión.

Antes de adentrarnos a examinar parte del caudal de acepciones modernas construidas en torno al conocimiento, es válido advertir que se está ante una discusión de trayectoria histórica, ya que desde tiempos de la antigua Grecia, los hombres se han preguntado por la manera como se genera el conocimiento, tanto en la experiencia personal como de forma amplia del denominado conocimiento científico.

La cuestión ha evolucionado desde la concepción de Platón en torno a la existencia de un mundo de las ideas externo y que por tanto deviene al hombre. De allí, ingresará la preocupación por discernir entre lo verdadero y no verdadero o aparente. En otros momentos, se considerará la experiencia sensorial y de allí la discusión se tornará más hacia el uso de la razón y la subjetividad del ser. Con el advenimiento del conocimiento científico, la cuestión adquirirá otro matiz, ya que el interés por explicar y prescribir, para algunos, y comprender para otros, atizará el asunto orientándolo aún más hacia el método y el sustento de lo que es verdadero y objetivo. Incluso la pugna conducirá a la censura o al desconocimiento de otras formas de conocer. Finalmente, con la preponderancia de la empresa en la sociedad, el problema se particularizó en procura de comprender la manera como se crea el conocimiento organizacional. 
En la Modernidad diversos pensadores se refirieron al conocimiento ${ }^{2}$. Bacon sostuvo que éste es el producto de la experiencia y que es a su vez poder. En esta misma línea, Hume planteó que ningún objeto que se descubra por las cualidades tal cual aparecen a los sentidos, ni las causas que lo produjeron o los efectos que se derivan de ellas, ni puede nuestra razón sin la ayuda de la experiencia hacer inferencias acerca de la existencia real. También Kant reconoce que todo conocimiento comienza por la experiencia, sin que todo proceda de la experiencia. En Kant, el conocimiento está compuesto por partes distintas y separadas: intuiciones y concepto. Así, el conocimiento para Kant será en cada caso un proceso de síntesis trascendental. En Husser, es la apertura intencional de un sujeto a un objeto presente. Por su parte Polanyi sostiene que el conocimiento es tácito o tiene sus raíces en el conocimiento tácito. En Habbermas, se advierte al conocimiento como un producto intencional $\mathrm{y}$ algunas veces inconsciente, por tanto como entidad no abstracta. Finalmente, Morin lo definirá como un fenómeno multidimensional e inseparable en el que confluye lo físico, biológico, cerebral, mental, psicológico, cultural y social.

Antes de adentrar en mayor detalle acerca del conocimiento hay que advertir que todos los hombres por naturaleza se orientan hacia el conocimiento. Tal vez como una manera innata para sobrevivir, desde que nace, el hombre busca conocer. Segundo, la forma primaria de conocimiento se sustenta en los sentidos, en la experiencia sensorial. Tercera instancia, la perspectiva objetivista conducirá el asunto hacia la existencia de una realidad externa e independiente del sujeto que pasada por la experiencia deviene en conocimiento. Así mismo, las consideraciones pragmatistas sostendrán que lo real se define en relación con lo útil, lo que funciona. Frente a estas consideraciones se planteará un reclamo por la exclusión del hombre y de la sociedad, situación que se manifiesta desde las ideas interpretacionistas expuestas por Nietzche y Heidegger, así como por los hermenéuticos, fenomenólogos, entre otros.

En este mismo sentido, la profesora Luz Marina Rivas (2013), al reflexionar sobre el tema enmarca al conocimiento como algo inherente al ser, como unidad entre sujeto, objeto, mundo. Se trata de una relación indisoluble que existe entre el sujeto que conoce, el objeto por conocer y el mundo en que se encuentra. Para la profesora Rivas, el conocimiento es más que una respuesta de un sujeto a su instinto de supervivencia, es la manera como la especie humana se adapta ante las presiones del mundo hostil ${ }^{3}$.

Así mismo, vale advertir que existe pleno consenso en cuanto a la diferencia existente entre lo que es el dato, la información y el conocimiento. De acuerdo con Newman (1997), los datos son una serie de números o caracteres carentes de significado por sí mismos. El análisis de los datos y su contextualización se convierten en información. La interpretación de la información se transforma en conocimiento útil. En este mismo sentido, Bohn (1994) establece que los datos son los que se advierten directamente por el sistema sensorial, la información son datos organizados o dentro de un contexto y por tanto dotados de significado, en tanto que el conocimiento conduce a la realización de predicciones, asociaciones causales o decisiones prescriptivas acerca de qué hacer.

Siguiendo a Sveiby (1997), se tiene que a diferencia de lo que sucede con la información, el conocimiento es intrínseco a las personas. El conocimiento ocurre, por tanto, como parte del proceso de interacción entre las mismas. La información per se tiene poco valor y sólo pasa a ser conocimiento cuando es procesada por el cerebro humano. Situación acogida también por Chinying (2001):

2 Nuestros agradecimientos a la profesora Luz Marina Rivas, quien generosamente nos transfirió sus reflexiones escritas aún sin publicar en torno al conocimiento. Para los propósitos de esta reflexión, se transcribieron de allí las ideas de este párrafo.

3 Rivas, L. M. Reflexión en torno al concepto de Conocimiento (inédito). Universidad Eafit. Medellín. 2013. 
El conocimiento es diferente de la mera información ya que el conocimiento es un acto humano...es el residuo del pensamiento... creado en el momento presente...pertenece y circula a través de las comunidades...en contraste con la información almacenada...la persona que conoce tiene información acerca de algo y capaz de utilizar esa información para resolver problemas. (p. 545).

De acuerdo con Nonaka (2005) citado por Orrego (2012, p. 205) el conocimiento es creado a través de la interacción dinámica entre lo subjetivo (que emerge del contexto imbricado por los actores sociales) y la objetividad del proceso social de validación del conocimiento. Por esta razón, Orrego encuentra una relación de transitividad entre el conocimiento como subcategoría y la innovación del emprendedor. Para Nonaka y Krogh (2009) el conocimiento es tácito y la creación de conocimiento es un proceso de va de lo tácito a lo explícito y de lo individual a lo social, y viceversa; formando, metafóricamente, una espiral.

Bajo el lente de la creación del conocimiento organizacional, la teoría defineel conocimiento en tres partes: primero, comouna creencia verdadera justificada, es decir, las personas justifican sus creencias sobre la base de sus interacciones con el mundo. Segundo, el conocimiento es la realidad de la acción hábil y/o potencial de definir una situación a fin de permitir la acción. En tercer lugar, el conocimiento es explícito y tácito a lo largo de un continuo. La "piedra angular" de éste cometido será el conocimiento tácito, el cual comprende los conocimientos atados a los sentidos, habilidades, movimientos, intuición y experiencias físicas; en pocas palabras lo que reside en la mente y el cuerpo humano (Nonaka y Krogh, 2009).

EnArrow (1962)laadquisición deconocimiento se remite al aprendizaje, el cual es producto de la experiencia y solo puede tener lugar a través del intento de resolver un problema, por lo que sólo tiene lugar durante la actividad. Así mismo, Bohn (1994), refiriéndose al conocimiento tecnológico, es decir, el de cómo producir bienes y servicios, establece que éste (el conocimiento) es incompleto y se desarrolló poco a poco a través de diversos tipos de aprendizaje. Es meritorio destacar como Gehani (2002) al realizar el análisis de Chester Barnard en "Las funciones del ejecutivo" encuentra que Barnard establece dos grandes categorías del conocimiento: el teórico, adquirido en la educación formal y el intuitivo personal y local. Lo esencial del asunto en Barnard, es la preponderancia dada al conocimiento intuitivo el cual considera es indispensable para el cumplimiento de todas las acciones del ejecutivo.

El caso de los empresarios de la actividad acuícola del Huila: ¿los podemos llamar empresarios innovadores?

A continuación se desarrolla el caso de dos agentes económicos de la actividad acuícola en el Huila, no sólo pioneros de la actividad sino los mayores productores, de cuyo relato se inferirá la discusión propuesta en el inicio del presente artículo de investigación. Primero se hará un esbozo del entorno de actuación de los agentes, para luego relatar, en forma sintética, los casos escogidos.

El contexto de los agentes: El departamento del Huila a finales del siglo XX

El Departamento del Huila se encuentra ubicado al sur de Colombia y su capital es la ciudad de Neiva. Es una región de tradición pastoril y agraria, calificada como periférica y de tardío desarrollo que no llegaría a incorporar procesos de desarrollo capitalista empresarial significativos y urbanos sino a partir de la década de 1930 con el despliegue de la agroindustria en torno al cultivo del arroz y café, así como una economía de servicios y comercio.

Con una población ligeramente superior al millón de habitantes (DANE, 2008) y una economía sustentada en el comercio, los servicios y los sectores agropecuario y piscícola, el departamento del Huila se presenta hoy en el contexto nacional como una región líder en producción piscícola de Tilapia, primer productor de café, quinto productor de petróleo y con inversiones dinámicas en la producción de energía eléctrica. 
Los acuerdos de competitividad impulsan en el siglo XXI al departamento hacia la agroindustria de base tecnológica sostenible en cafés especiales, frutales, cacao y tabaco; el fomento de la industria del turismo, la consolidación de la cadena piscícola, la industrialización de los fosfatos, la arcilla y el mármol, y finalmente la generación de energía eléctrica.

En particular, se advierte que la piscicultura en Colombia se practica desde la década de 1940. Sin embargo, será tan sólo en las década de 1980 que se desarrollen proyectos importantes con la ayuda de agencias de cooperación internacional. En 1984 se establece en el departamento del Huila la primera experiencia significativa en el cultivo de Tilapia Roja Nilótica. Se trató de una empresa de origen familiar instalada en la hacienda "Castalia", cultivo., que aunque modesto, comparado con la producción actual, se establecía como el único en producir en forma organizada.

Con el incremento en el consumo per cápita de la Tilapia y la conformación de un mercado local, se inició en los noventa una década con buenos augurios para esta actividad. El proceso de apertura estimuló el crecimiento de la demanda lo que significó iniciar las exportaciones hacia Estados Unidos. La actividad pasó de producir 3.040 toneladas en 1991 a 11.050 en el año de 1992. Esta dinámica hizo que el estado creara a inicios de la década un instituto especializado para la pesca y la acuicultura (INPA). En el ámbito internacional, se advertían similares iniciativas en países como Venezuela, Honduras, Costa Rica y Jamaica.

Con la asesoría de expertos internacionales se dio uso a los "residuos" del proceso como cabezas, espinazos, recortes y la piel de la tilapia. En 1993 fue fundada en el Valle del Cauca la empresa Camarpez destinada a la curtiembre de la piel de la tilapia. La producción se afianzó al punto que para el año de 1995 se produjo 16 mil toneladas de tilapia, doblando la producción de camarón y triplicando la de trucha y cachama (Inpa, 1997).

El crecimiento de la demanda interna del producto y el cierre de la principal empresa Colapia S.A en 1997, estimuló la puesta en marcha en proyectos de cultivo de tilapia bajo la modalidad de jaulas flotantes en los embalses de los departamentos del Huila, Cauca y Tolima. De esta manera, la producción de tilapia se aproximó a las 20 mil toneladas en 1999, superando esta cifra a partir del año 2000 (Castillo, 2003).

Precisamente, será la década del año 2000 en la que se intensificará el cultivo de la tilapia en jaulonesyconelusodeabundantescuerposdeagua existentes en los embalses. El departamento del Huila se posicionará como líder de la producción nacional de tilapia, logrando una producción cercana a 16 mil toneladas, equivalentes al 30\% de la producción nacional (Gobernación del Huila, 2013), destinadas mayormente al consumo del mercado local, con exportaciones menores al mercado norteamericano.

De acuerdo con un informe de la gobernación del departamento (Gobernación del Huila, 2013) el Huila se consolidó en la década del año dos mil primer productor nacional de tilapia aportando el $53 \%$ de la producción total, lo que generó alrededor de 5.288 empleos directos con ingresos por exportaciones anuales de 5.5 millones de dólares. En la actualidad coexisten tres tipos de productores: 1) pequeños productores que generan cerca del $20 \%$ de la producción cuyo mercado es el intermediario; 2) los medianos productores, que producen por encima de 80 toneladas por año y aportan el $25 \%$ de la producción y 3) los grandes productores con volúmenes de producción superior a las 800 toneladas por año, produciendo alrededor del $60 \%$ del total de tilapia. Este grupo está integrado por 9 empresas que integran toda la cadena productiva, es decir, producen desde el alevino hasta el proceso industrial del filete.

Tal como se advierte, la dinámica apreciada en el departamento del Huila en cuanto a la creación de organizaciones empresariales formales, insertas en la lógica del mercado acuícola es reciente. La primera de ellas, data de 1984. Sin embargo, fue en la década de los noventa que inició la dinámica conocida hoy en día. Cinco de las nueve empresas calificadas como gran productor fueron establecidas en la citada década. Dada la importancia que registran estas organizaciones 
en el contexto local y nacional, así como la particularidad del fenómeno y su manifestación reciente sin ningún referente en la localidad, se hace atractivo comprender tal manifestación.

\section{Pioneros de la actividad piscícola en el Huila}

\section{Carlos Cabrera Villamil ${ }^{4}$}

Oriundo del municipio de Aipe y líder del sector piscícola. A pesar de la pérdida de su padre a la edad de 7 años, hereda de él la pasión por el trabajo, la tecnología y el desarrollo de proyectos productivos en el sector agropecuario. Cursó estudios de primaria y secundaria en el internado del colegio Calasanz de Colombia, donde desarrolló valores y hábitos como la disciplina, respeto, autonomía y el deporte.

Desde temprana edad asumió responsabilidades como administrador de una finca familiar y posteriormente incursionó como productor y comercializador de frijol, sorgo y arroz. Por solicitudes de gobernadores en dos oportunidades desempeñó cargos públicos. El primero como alcalde de Aipe y luego como Secretario de Fomento Agropecuario y Minero, cargo que le permitió impulsar actividades agropecuarias de alto impacto en el Departamento. Como Secretario de Fomento tuvo la oportunidad de identificar el potencial del Departamento para la actividad piscícola, labor a la que posteriormente le dedicaría todos sus esfuerzos, aprovechando los beneficios que ofreció la Ley Páez.

Inició su actividad empresarial con jaulas flotantes en la Represa de Betania y posteriormente incursionó con la producción en lagos artificiales, en donde pone a prueba su capacidad para capitalizar la transferencia de conocimientos de expertos en manejo de aguas y la aplicación de genética en la producción de alevinos. En 1997 crea la empresa 3C y en el 2000 la empresa Pacandé, hoy reconocidas en el mercado nacional por la capacidad de producción y la tecnología utilizada en las diferentes etapas de la cadena de valor.

Tabla 1. Dinámica de la evolución empresarial

\begin{tabular}{|l|l|l|}
\hline \multicolumn{3}{|c|}{ DINAMICA DE LA EVOLUCION EMPRESARIAL } \\
CARLOS CABRERA VILLAMIL
\end{tabular}

Fuente: Construcción propia.

La tabla número 1 nos indica que la constitución de Carlos Cabrera como pionero de la actividad acuícola es el resultado de un proceso evolutivo, en el que se hereda no sólo un capital económico sino de conocimientos y capacidades transmitidas en el seno de su familia. Capital familiar que se incrementa con la experiencia ampliada en la gesta agropecuaria, la comercialización, los vínculos gremiales y la administración pública, para citar algunos.

\section{Eugenio Silva}

Desde muy temprana edad fue influenciado por las actividades agropecuarias y ganaderas. Realizó estudios secundarios en Bogotá y cursó Administración de Empresas de la Universidad del Rosario.

Durantesujuventud compartiójuntoasu padre experiencias en la captura de peces ornamentales y pesca de bagre en el departamento del Caquetá,

4 Una versión más ampliada de los empresarios reseñados se encuentra en los productos obtenidos dentro del proyecto denominado "Origen de la actividad acuícola en el Huila, 1985-2005", desarrollado por los autores con el auspicio de la Universidad Surcolombiana, en el cual se reseñó tanto la historia de la actividad como la historia de vida de los empresarios pioneros. 
labor que posteriormente por inseguridad y confrontación armada en la zona, tuvo que abandonar. La experiencia en comercialización de pescado fue fundamental en su proceso formativo como empresario.

Consolidó su formación en el área de mercadeo cuando se vinculó como coordinador de equipos de ventas primero con el grupo Carvajal y luego el Diario El Espectador. Impulsó la creación de la empresa "Hielo Artico" junto a sus hermanos. La citada empresa atendía parte del mercado de Bogotá. Tras su retorno a Neiva crea una empresa piscícola con la técnica de jaulas flotantes en guadua en la represa de Betania. Gradualmente desarrolla salas de proceso y transporte especializado. Incursiona en el mercado internacional y diversifica las inversiones con la creación de una fábrica de empaques en icopor. Su liderazgo, experiencia y conocimientos del sector piscícola lo llevan a liderar la Asociación de Piscicultores del Huila.

La tabla número 2 muestra el papel que jugó en la conformación de un agente empresario la experiencia familiar en una actividad específica, la experiencia ocupacional laboral, la apropiación de nociones asociadas a la comercialización.

Tabla 2. Dinámica de la evolución empresarial

\begin{tabular}{|l|l|l|}
\hline \multicolumn{3}{|c|}{ DINAMICA DE LA EVOLUCION EMPRESARIAL } \\
EUGENIO SILVA
\end{tabular}

Fuente: Construcción propia
¿Hay algo nuevo, postschumpeteriano, en la teoría de la innovación?

A partir de la revisión efectuada tanto del concepto schumpeteriano de innovación como a las modernas o posteriores aportaciones teóricas, se detectan, al menos, tres aspectos que en su orden otorgarían una nueva dinámica para el análisis del empresario innovador:

1. La innovación tiene como sustento el conocimiento. No puede ocurrir sin éste. Por lo tanto, implica interacción del sujeto innovador para acopiar, mezclar, reutilizar, combinar y crear conocimiento. El conocimiento se plasma en la mente y el cuerpo del sujeto innovador, en sus rutinas y en las relaciones con su mundo.

La innovación depende del conocimiento y éste es el resultado de las experiencias en el sentido de las interacciones vividas por el innovador. Conocer no es sólo un proceso individual y subjetivo que se limita a la asimilación de un conjunto de situaciones o experiencias. También entra en juego la interpretación que se abastece de significados y comprensión, los cuales a su vez se construyen. En suma, es un proceso activo y socialmente contenido.

2. La innovación es la creación e introducción de algo nuevo con aceptación. El sujeto innovador como productor de nuevas realidades. Nos referimos a unos sujetos empresarios situados en unas condiciones particulares de existencia que lo condicionan, que lo llevan a plantear unas prácticas para la creación e introducción de nuevas realidades que garanticen su subsistencia.

3. La innovación es un proceso ampliado (abierto). Esto implica el ingreso o el acceso a unos insumos de conocimiento ubicados en el innovador y en partes externas a él, en su espacio social. Implica ubicar y establecer al innovador en un entramado de relaciones (red), integrarlo a ellas. También implica unos mecanismos de integración y transformación de tales insumos, es decir adelantar acciones, un conjunto de prácticas conducentes a la transformación y por supuesto a la reproducción de algo, de otra realidad. 
Lecciones para una historiografía del empresario: ¿Cómo dos agentes dedicados a diversas actividades empresariales pueden dar origen a una actividad económica? ¿Se pueden catalogar como empresarios innovadores?

La realidad social y por tanto empresarial es compleja y no se puede reducir a un solo aspecto. Sólo para efectos de los propósitos del presente escrito, abordaremos el análisis desde la particularidad del conocimiento y la innovación. Siendo así, se tiene que la primera situación advertida en los empresarios analizados es la apertura hacia la actividad empresarial a partir de la apropiación de capital de conocimiento tradicional, instalado, por un parte, en el entorno familiar con la producción ganadera (caso Carlos Cabrera Villamil), y por la otra, en la cultura, en cuanto vocación económica hacia la actividad pesquera por parte de un vínculo paterno, lo que se suma a la apropiación del conocimiento a partir de la interacción en la experiencia ocupacional (caso Eugenio Silva). Entonces, entre otras cosas, estos agentes se inician en la gesta de unidades de acumulación económica a partir del conocimiento técnico, de un saber hacer.

Sorprende que en los dos casos, la inversión se especialice, estableciendo un portafolio para sus inversiones. La conformación de sociedades es el medio como los sujetos en análisis llegaron a involucrarse en actividades diversas y de nula o escasa experiencia en las cuales se fueron especializando. En la práctica se aprecia la integración del capital económico con el conocimiento.

En el caso del primer empresario, Carlos Cabrera Villamil, cierra su ciclo como empresario en actividades distantes de su experiencia inicial, es decir, agropecuarias. De extracción primaria asociada a las actividades ganaderas y de producción de frijol, sorgo y arroz se continuó con la producción, transformación y comercialización piscícola.

En cuanto al segundo empresario, Eugenio Silva, no hay duda su inclinación desde muy joven por las actividades asociadas a la pesca; no obstante, la acumulación de conocimiento y experiencias en el área de las ventas, trabajo en equipo y liderazgo como empleado de empresas nacionales, así como la formación profesional como Administrador de Empresa y la incursión como socio de una empresa de producción de hielo en Bogotá, lo llevaron posteriormente a retomar la apuesta por la piscicultura.

Con todo ello se puede colegir que la puesta en marcha de unidades empresariales por parte de los sujetos estudiados demandó conocimiento, especialmente, técnico. Este conocimiento no se recuperó de una base de datos que informara las condiciones del mercado, ni de un plan de negocios. Sino que fue elaborado y reelaborado activamente en el contexto del negocio mismo y del potencial empresario; donde él se posibilitó, se construyó, donde él fue actor; en un tiempo y un espacio específico. El conocimiento se estructura en una interacción de actores que comparten mensajes. El medio habla y el receptor crea sentido, interpreta.

El conocimiento posee en si mismo un valor histórico atribuido por las aportaciones $\mathrm{o}$ transferencias que hicieron por mucho tiempo, como en los casos analizados, las comunidades, la familia y las sociedades de negocios. El conocimiento se crea, circula y se enriquece en el medio social al pasar el tiempo.

¿Se pueden catalogar como empresarios innovadores los agentes estudiados?

La revisión a Schumpeter indica que él diferencióla innovación radical de la incremental. Cuando planteó las nociones de crecimiento económico y desarrollo económico, asoció el cambio o el paso del primer estado al segundo como un resultado de las innovaciones radicales causantes de tal desequilibrio. Si nos atenemos a esta diferencia sustancial América Latina quizá no tendría empresarios innovadores.

La situación anotada, está definida por la relación con los desarrollos científicos y tecnológicos aplicados, causantes de desequilibrios sociales y económicos. Es decir, la innovación vista en los empresarios estudiados es básicamente incremental, por adaptación tecnológica y la 
realización de mejoras. Ahora, eso no significa que nuestros agentes tengan menos valía. Precisamente, esas prácticas que conllevan a innovaciones incrementales reflejan una realidad por descubrir, o sea la lógica de actuación de esta clase social. Dar cuenta de la práctica de los empresarios significa precisar sus procesos, adaptaciones, recontextualizaciones, mejoras, cambios, trayectorias y disposiciones. En el siguiente acápite profundizará en éste asunto. Estamos en el momento para diferenciar una historia de empresarios de una empresarial.

Para la discusión: Réplica a la inclusión del empresario schumpeteriano en el análisis historiográfico

En la innovación está presentela acción humana. Es el resultado de ésta. En toda acción humana están presentes elementos que no se pueden analizar en términos pragmáticos de resultados, tal como la innovación, sino de prácticas, acciones organizadas de los sujetos para construir una realidad, vale decir, su realidad.

El empresario y la innovación se pueden comprender en mejor manera por las prácticas de los sujetos en un entramado. De la misma forma que las innovaciones radicales no bastan para comprender la acción humana, tampoco el proceso de innovación se puede comprender sin las prácticas y las interacciones de los sujetos innovadores.

Si elevamos la discusión a calificar o catalogar agentes económicos para establecer si son o no empresarios, no da lugar a un proceso de comprensión. Para que surja el proceso de innovación se requiere abandonar el resultado y observar la manera como los agentes interactúan y construyen esta realidad.

Nuestra opinión es que una visión analítica de unos agentes económicos, actuando de manera independiente y separada de su realidad, constituye algo peligrosamente incompleto. Precisamente, el énfasis puesto en los resultados, es decir, si obtuvo innovación y si ésta fue o no radical o incremental, o si tiene validez que sea de producto, proceso $u$ organizativa, ha dado lugar a una noción de empresario como un prohombre que actúa por fuera de unas posibilidades, de unas proximidades, que lo enmarcan, que lo constituyen y desde las cuales produce realidad.

Esta noción de empresario innovador, como un individuo racional y aislado, es culpable de ese reducido y exclusivo énfasis sobre "el producto terminado". No nos dice nada de cómo entretejer el tramado para posibilitar la construcción de realidad, para la construcción del empresario y la idea del negocio. Unos individuos que aparecen ya "hechos y acabados", actuando de manera racional y aislada, sin que medien o se introduzcan estructuras y mecanismos para las elecciones y reproducción de éstos mismos; no aportan verdaderas respuestas a una sociedad.

La percepción teórica desde la perspectiva de Schumpeter de la innovación como un fenómeno eminentemente individualista, deja de lado concebir la innovación como un resultado de social e inmerso en las estructuras sociales y redes sociales; en tal sentido, los innovadores no realizan sus actividades de manera aislada, por el contrario se encuentran conectados a redes de acceso al poder, la información, el conocimiento $\mathrm{y}$ los recursos financieros.

En vez deinsistiren calificar agentes económicos de innovadores o no, sería muy útil establecer el proceso de creación del conocimiento y su transformación en innovación para la realidad empresarial. También sería justo, construir una categoría más amplia de empresario como un sujeto en acción, como un sujeto con prácticas, un sujeto que construye realidad. Las nociones de Pierre Bourdieu, tales como clase social, campo, capital, hábitus, estrategias de reproducción, entre otras, son tan útiles para los investigadores en ciencias sociales, como otras nociones de combinación de recursos, riesgo, incertidumbre, detector de oportunidades e innovador, pueden serlo para los economistas.

Los conceptos de Bourdieu, tal como el de reproducción social, rescata al agente social quien se reproduce, señala principios de explicación y comprensión como resultado simultáneo y dialéctico de las condiciones objetivas de vida, externas e incorporadas. Así, las estrategias del 
agente social (el empresario y la innovación) se explicarían dentro del contexto del sistema que constituyen y dentro del espacio social global, donde las prácticas que forman parte de ese sistema se relacionan con las prácticas constitutivas de los demás, articulando modos de reproducción sociales diferenciales (Gutiérrez, 2001).

Los conceptos propuestos desde Bourdieu, incluso, no restringen el análisis de la producción de la innovación, cosa que si sucede al trasladar simplemente la categoría schumpeteriana. El empresario innovador de Schumpeter es un agente que combina unos medios dados y adaptados a un fin, para la introducción de lo resultante de la nueva combinación. Este concepción presupone unos medios "dados", que surgen porque sí, que están simplemente allí. Tal como se describió anteriormente, la innovación, como los empresarios, surge en la interrelación, en el entramado, es un proceso.

Entonces, ¿De qué vamos a ocuparnos en la historiografía empresarial? ¿Qué reclama la sociedad y las instancias académicas que los historiadores puedan proponer en relación con los empresarios?

\section{Referencias}

Arrow, K.J. (1962). The economics implications of learning by doing. Review of Economic Studies, 29(6), p. 155-173

Arroyo, J.H. (2006). Historia de las prácticas empresariales del Valle del Cauca. Cali 1900-1940. Cali: Universidad del Valle.

Bohn, R.E. (1994). Measuring and managing technologicalknowledge. Sloan Management Review, 36(1), p. 61-73.

Bigliardi, B. and Galati, F. (2013). Models of adoption of open innovation within food industry. Trends in Food Science \& Technology, 30 p. 16-26.

Chesbrough, H. (2003). The era of open innovation. MIT Sloan Management Review, $44(3)$, p. 35-41.
Chinying Lang, J. (2001). Managing in knowledge-based competition. Journal of Organizational Change, 14(6), p. 539-552.

Dane (2008). Proyección de la población 2006-2020. Recuperado el 12 de diciembre de 20013, de: http://www. dane.gov.co/files/investigaciones/ poblacion/proye pobla06_20/ MProyeccionesMunicipalesedadsexo.pdf

Dávila, C. (1986). El empresario colombiano, una perspectiva histórica. Bogotá: Pontificia Universidad Javeriana.

Dávila, C. (2003). Empresa y empresarios en la historia de Colombia. Siglos XIX y XX. Bogotá: Grupo Editorial Norma.

Djankov, S., et al (2005). Who Are Russia's Entrepreneurs? Journal of the European Economic Association, 2005 (Papers and Proceedings), 3(2-3), pp. 587-97.

Djankov, S., et al. (2006). Who Are China's Entrepreneurs? American Economic Review, 96(2), 348-352.

Dtling, F., Lehner, P., and Trippl, M. (2006). Innovation in Knowledge Intensive Industries: The Nature and Geography of Knowledge Links. European Planning Studies, Vol. 14, No. 8, p. 1035-1058.

Gehani, R.R. (2002). Chester Barnard's "executive" and the knowledge-based firm. Management Decision, 40(10), p. 980-991.

Groenewegen J., and Van der Steen M. (2006). The Evolution of National Innovation Systems. Journal of Economic Issues, 60 (2), p. 277-285.

Gutiérrez, A. (2011). Clases, espacio social y estrategias. Una introducción al análisis social de la reproducción social en Bourdieu. En Pierre Bourdieu, Las estrategias de la reproducción social. (pp. 9-27). Buenos Aires: Siglo XXI editores. 
Kirzner, I. (1979). Perception, opportunity, and profit. Chicago: Chicago University Press.

Kirzner, I. (1998). El empresario. Competencia y empresarialidad. Madrid: Unión Editorial

Knigth, F.H. (1947). Riesgo, incertidumbre y beneficio. Madrid: Aguilar.

Kogut, B. (2000). The network as knowledge: generative rules and the emergence of structure, Strategic Management Journal, 21(3), p. 405-425.

Lundvall, B. A. (1996). “The social dimension of the learning economy", en Danish Resarch Unit for Insdustrial Development, Working Paper $\mathrm{N}^{\circ}$ 96-1, 24.

Lundvall, B.A., et al. (2002). National systems of production, innovation and competence building, Research Policy, 31(2), p. 213-231.

Molina, L. F. (1988) Empresarios Colombianos del siglo XIX. Bogotá: Banco de la República. Ancora Editores.

Molina, L. F. (2003). Francisco Montoya Zapata. Poder familiar, Político y empresarial 18101862. Medellín: Nutifinanzas.

Newman, V. (1997). Redefining knowledge management to deliver competitive advantage. Journal of Knowledge Management. Vol. 1, núm. 2, p. 123-132.

Nonaka, I., von Krogh, G., (2009). Tacit knowledge and knowledge conversion: controversy and advancement in organizational knowledge creation theory», Organization Science, 20(3), p. 635-652.

OEDC/European Communities (2005). Manual de Oslo: Guía para la recogida e interpretación de datos sobre innovación. Madrid. 3d. Ed., OECD/EC.

Paré, S., \& Therasme, K. (2010). Entrepreneurs in the new economy: Immigration and sex effects in the Montreal metropolitan area. Journal of International Entrepreneurship, 8(2), 218-232.
Quintero, A. y Centeno R. (2007). Hacendados, Comerciantes y Negociantes de Neiva a finales del siglo XIX. Grupo de investigación Pymes. Neiva: Universidad Surcolombiana.

Ranga, L.M., Miedema, J., Jorna, R. (2008). Enhancing the innovative capacity of small firms through triple helix interactions: Challenges and opportunities, Technology Analysis \& Strategic Management, 20(6), p. 697-716.

Ross, J. and Von Krogh, G. (1996). The epistemological challenge: managing knowledge and intellectual capital. European Management Journal, 14(4). p.333-337.

Safford, F. (1977). Aspectos del siglo XIX en Colombia: significación de los antioqueños en el desarrollo económico colombiano. Bogotá: Universidad Nacional.

Safford, F. (1989). El ideal de lo práctico. El desafío de formar una élite técnica y empresarial en Colombia. Bogotá: Universidad Nacional.

Schumpeter, J.A. (1997). Teoría del desenvolvimiento económico. México. Fondo de Cultura Económica.

Schumpeter, J.A. (2002). Ciclos económicos: análisis teórico, histórico y estadístico del proceso capitalista. Zaragoza: Prensa universitaria.

Sveiby, K. E. (2001) A knowledge-based theory of the firm to guide in strategy formulation. Journal of Intellectual Capital 2(4), p. 344-358.

Taylor, W. (1990). The business of innovation: an interview with Paul Cook, Harvard Business Review, 68(2), p. $96-110$.

Zen, A., \& Fracasso, E. (2008). Quemé é o Empreendedor? As Implições de Três Revoluções Tecnológicas na Construção do Termo Empreendedor. (Portuguese). Revista De Administração Mackenzie, 9(8), p. 135-150. 